\title{
Correlation of Phosphorus Cross-linking to Hydration Rates in Sodium Starch Glycolate Tablet Disintegrants using MRI
}

Anuji Abraham ${ }^{1 *}$, Dolapo Olusanmi ${ }^{1}$, Andrew J. Ilott ${ }^{2}$, David Good ${ }^{1}$, Denette Murphy ${ }^{1}$, Daniel Mcnamara $^{1}$, Alexej Jerschow ${ }^{2}$ and Rao V. Mantri ${ }^{1}$

${ }^{1}$ Bristol-Myers Squibb, Materials Science and Engineering, Drug Product Science and Technology, New Brunswick, New Jersey 08903, USA.

${ }^{2}$ New York University, Department of Chemistry, New York, New York 10003, USA.

\section{KEYWORDS}

FLASH MRI, Infrared spectroscopy, Tablet disintegration, Tablet imaging, Disintegrants, SSG, Sodium starch glycolate, Imaging methods

*Corresponding author 


\begin{abstract}
Understanding the behavior of tablet disintegrants is valuable in the development of pharmaceutical solid dosage formulations. In this work, high-resolution Magnetic Resonance Imaging (MRI) has been used to understand the hydration behavior of a series of commercial sodium starch glycolate (SSG) samples, providing robust estimates of tablet disintegration rates that could be correlated with physicochemical properties of the SSGs, such as the extent of phosphorus cross-linking as obtained from infra-red spectroscopy. Furthermore, elemental analysis together with PXRD has been used to quantify the presence of carboxymethyl groups and salt impurities, which also contribute to the disintegration behavior. The utility of Fast Low Angle SHot (FLASH) MRI has been demonstrated as an approach to rapidly acquire approximations of the volume of a disintegrating tablet and, together with a robust voxel-analysis routine, extract tablet disintegration rates. In this manner, a complete characterization of a series of SSG grades from different sources has been performed, showing the variability in their physicochemical properties and demonstrating a correlation between their disintegration rates and intrinsic characteristics. The insights obtained will be a valuable aid in the choice of disintegrant source as well as in managing SSG variability to ensure robustness of drug products containing SSG.
\end{abstract}




\section{Introduction}

Disintegrants are excipients included in pharmaceutical solid dosage formulations such as tablets and capsules to help the tablet to break apart in the digestive tract. Their purpose is to facilitate dissolution by breaking down the tablet into smaller fragments to ensure that it is in contact with the dissolution medium. The most widely used disintegrants include cross-linked polymers such as polyvinylpyrrolidone (crospovidone) and sodium-carboxymethyl cellulose (croscarmellose sodium) and also modified starch such as sodium starch glycolate (SSG). ${ }^{1-3}$ The disintegration properties depend on the many different chemical attributes of the disintegrant, such as the extent of cross-linking, hydroxylation and carboxymethylation. The cross-linking reduces the solubility in water and limits the viscosity of the surrounding water, thus achieving better release of the drug. Hydroxyl groups in these compounds lead to strong hydrogen bonded networks that can reduce water access into the polymer. However, when these groups are partially substituted with carboxymethyl or similar hydrophobic groups the formation of hydrogen bonds is disrupted, allowing water access into the polymer. Some of these materials also contain salt impurities such as sodium chloride $(\mathrm{NaCl})$ and/or sodium citrate, which also allow rapid access of water into the polymer, facilitating dissolution and forming hydration spheres around the ions. It is thought that an optimum balance between carboxymethylation and cross-linking allows for rapid water uptake and swelling without resulting in a high viscosity that would delay dissolution. ${ }^{4}$ However, there is still not a clear understanding of the mechanism of water uptake into these polymeric materials. Meanwhile, the role of salt impurities, which is intuitively a hydrophilic effect, is not well studied. Understanding the water uptake and disintegration rates of SSGs in correlation to their physicochemical characteristic can help in choosing the right disintegrant for a given formulation.

This study focuses on understanding the physicochemical characteristics of sodium starch glycolate (SSG), such as the extent of cross-linking, ionic strength and hydrophilicity, and correlates these properties with the tablet disintegration rates. SSG is considered to be a super disintegrant and consists of potato starch cross-linked by phosphate groups that help to reduce the water-soluble fraction of the molecule. The interactions with water are further modified by the partial substitution of the hydroxyl protons with carboxymethyl groups, making carboxymethyl ether groups, which disrupt hydrogen bonding and allowing water access into the polymer. The United States Pharmacopeia (USP) and 
European Pharmacopoeia (Ph. Eur.) classify SSGs according to their extent of sodiumcarboxymethylation, into two types. Type A contains 2.8 - 4.2\% sodium, while Type B contains 2.0 $3.4 \%$ sodium, with a limit of $7 \% \mathrm{NaCl}$ impurities in both types. However, there is no mention of phosphate cross-linking or other salt impurities, such as sodium citrate, although these properties are important in tuning the behavior of SSGs as disintegrants and may control their performance in pharmaceutical formulations. Additionally, Ph. Eur. does not require any functionality tests for SSGs. UPS has three major mechanisms to describe the function of the various disintegrants which are volume increase by swelling, deformation, and capillary action (wicking), though there is no specific functionality test for SSG.

Swelling and hydration studies are usually performed visually to understand the water uptake and tablet disintegration mechanisms. ${ }^{5}$ However, information obtained visually ${ }^{5}$ is often limited, hard to quantify, and may differ from the microscopic information obtained by spectroscopic or imaging techniques. There are very few techniques capable of understanding the process of fast $(<10$ mins $)$ tablet disintegration in real-time, but one possibility is Magnetic Resonance Imaging (MRI). ${ }^{6-20}$ MRI is a versatile technique that can evaluate the full volume and cross section of tablets and the surrounding liquid into which they dissolve across a relatively large range of timescales. It has been widely used for monitoring tablet swelling over long time periods (usually a few hours) and for understanding the kinetics of extended release tablets. ${ }^{21}$ Quodbach et. al. has pioneered and been successful in studying the disintegration process of tablets using advanced real-time MRI techniques, particularly Fast Low Angle SHot (FLASH) sequences. ${ }^{6-8}$

The first part of this study focuses on a thorough analysis of a series of SSGs using Infra-red (IR) spectroscopy, elemental analysis and powder X-Ray diffraction (PXRD), giving insights into the variability of the physicochemical properties of SSGs. The second part of the study focuses on understanding the disintegration and water uptake rates of a series of commercial SSG compacts using high-resolution, real-time MRI and correlates these observations with the measured physicochemical properties of the excipients, such as the extent of cross-linking, carboxymethylation and salt impurities. The extent of phosphorus cross-linking is understood with the help of IR spectroscopy, while sodium citrate salt impurity levels are quantified using PXRD analysis, and $\mathrm{P}, \mathrm{Cl}$ and total $\mathrm{Na}$ content are quantified using elemental analysis allowing $\mathrm{Na}$ associated with either $\mathrm{NaCl}$ or the carboxymethyl groups to be ascertained. The FLASH MRI pulse sequence ${ }^{22}$ is used to view the full cross section of the 
tablet disintegration, resolving true disintegration processes. The quantification of the disintegration rates is done using an analysis of the full volume of the tablet at each time point.

\section{Experimental}

\section{Materials}

Five sodium starch glycolate samples (Type A) from different sources were investigated: Source A, Source B, Source C, Source D and Source E. As will be demonstrated, the different samples contain varying levels of phosphorus cross-linking, carboxymethyl groups and salt impurities.

\section{Tablet preparation}

The SSG compacts/tablets were precision made on an Instron Materials Testing System, model 5567, supplied by Instron USA, for compaction to within one hundredth of a millimeter for the target thickness. Each compact was individually weighed on an analytical balance to within one tenth of a milligram. Therefore, thickness and density were controlled to $<1 \%$ deviation between samples. For each SSG grade $80 \mathrm{mg}$ of material was compressed using a $3 / 16^{\text {th }}$ inch round flat faced F-type tooling at compression forces ranging from $\sim 1-3 \mathrm{kN}$ to achieve compacts with $4.763 \mathrm{~mm}$ diameter and $3.508 \mathrm{~mm}$ thickness. The goal was to achieve similar relative densities across all the grades. The in-die compact density was calculated to be $1.28 \mathrm{~g} \mathrm{~cm}^{-3}$.

\section{Magnetic Resonance Imaging (MRI)}

Experiments were performed on a Bruker Ultrashield 9.4 T Avance I spectrometer containing a Bruker Micro2.5 gradient assembly and operating at $400.13 \mathrm{MHz}$ for ${ }^{1} \mathrm{H}$. The images were collected using a Bruker Micro2.5 imaging probe equipped with a $15 \mathrm{~mm}{ }^{1} \mathrm{H}$ birdcage coil. A two dimensional Fast Low Angle SHot (FLASH) ${ }^{22}$ sequence was used with 10 interleaved sagittal slices and 1 coronal slice. The in-plane field of view (FOV) was $19.2 \times 19.2 \mathrm{~mm}$ with 96 points acquired in both the frequency and phase dimensions to give a nominal isotropic resolution of $0.2 \mathrm{~mm}$. The coronal slices were spaced with $0.25 \mathrm{~mm}$ between the slices, giving an overall coverage of $7.25 \mathrm{~mm}$, which was found to cover the full volume of the dissolution medium. The total echo time (TE) and repetition time (TR) were $1.4 \mathrm{~ms}$ and $50 \mathrm{~ms}$ respectively. The total time for each scan was ca. $10 \mathrm{~s}$. 
Experiments were started by adding $2 \mathrm{ml}$ of the dissolution medium (pH 2.8 citrate buffer) into a $10 \mathrm{ml}$ centrifuge tube placed inside the rf coil of the probe. The compact under study was then dropped into the centrifuge tube and the probe holding the sample was rapidly moved into the iso-center of the magnet and the MRI experiments were started. The delay between the start of dissolution and the acquisition of the first image was measured in a subset of experiments and was found to average between 10 and $15 \mathrm{~s}$ (for 1 or 2 people performing the experiment) and this time was accounted for in the time series. We note that all of the ${ }^{1} \mathrm{H}$ images acquired are observing the water protons in the dissolution media.

\section{Voxel analysis of MRI Results}

The aim of the analysis was to account for the full volume of the compacts at each time point in the experiment series in a manner that was immune to temporal image artifacts and variations in the image intensity caused by phenomena such as gel formation around the disintegrating tablet (apparent in the top series in Figure 1 as a dark region surrounding the tablet, corresponding to an area with decreased $T_{2}{ }^{*}$ compared to the pristine dissolution medium). This was achieved by creating a masked image, $M_{\mathrm{n}}^{\prime}$ by comparing each voxel in each slice of the acquired image, $M_{\mathrm{n}}$ to a threshold value, $t_{\mathrm{v}}$ :

$$
M_{\mathrm{n}}^{\prime}=\left\{\begin{array}{l}
1 \text { for }\left(M_{\mathrm{n}}<t_{\mathrm{v}}\right), \\
0 \text { for }\left(M_{\mathrm{n}}>t_{\mathrm{v}}\right) .
\end{array}\right.
$$

The effect of this transform on representative image slices is shown in Figure 1, where $t_{\mathrm{v}}$ was chosen to be $30 \%$ of the maximum image intensity. The figure illustrates that the artifacts from the coronal slice (vertical gray line in the middle of the image) and the gel formation are ignored by the analysis. This is a robust method to process a large amount of data semi-automatically. By applying this analysis to each slice of every image, a representation of the full tablet volume at each time point is acquired. The choice of $t_{\mathrm{v}}$ tunes the sensitivity to artifacts but the representation of the tablet volume is relatively immune.

\section{Fourier transform (FT) Infra-red (IR) spectroscopy}

FTIR spectroscopy was performed using a Nicolet Nexus 670 spectrometer with attenuated total reflectance (ATR). The spectra were collected using reflection mode with a resolution of $4 \mathrm{~cm}^{-1}$ and 64 scans. 


\section{Powder X-Ray diffraction (PXRD)}

PXRD patterns were recorded on a Bruker D4 Endeavor/Lynxeye (Bruker, Germany) X-ray powder diffractometer with $\mathrm{Cu} \mathrm{K} \alpha$ radiation: $\lambda=1.541 \AA$. The diffractometer was equipped with a ceramic tube that was set at the power level of $40 \mathrm{kV}$ and $40 \mathrm{~mA}$, and a PSD lynxeye detector. Incident optics consisted of a $0.6^{\circ}$ divergence slit. Diffracted optics consisted of Ni-K-Beta filter, and detector window of $3^{\circ}$. Data were collected in reflectance geometry, whilst spinning, over a $2 \theta$ range of $4-32^{\circ}$, with a step size of $0.03^{\circ}$, and counting time of $1 \mathrm{sec} / \mathrm{step}$ in continuous mode.

\section{Elemental analysis}

Elemental analysis of phosphorus (P) was performed using a nonspecific inductively coupled plasmaatomic emission spectroscopy (ICP-AES) method. Elemental analysis of chlorine $(\mathrm{Cl})$ and sodium $(\mathrm{Na})$ were done by Robertson Microlit Laboratories, New Jersey, USA. The inductively coupled plasmaoptical emission spectroscopy ICP-OES method was used to analyze $\mathrm{Na}$, while the titration method was used to analyze $\mathrm{Cl}$. Results provided will have $+/-20 \%$ error.

\section{Results and discussions}

The first part of this study aimed to show the variability in the physicochemical properties of the series of SSG grades by measuring these properties using IR spectroscopy, elemental analysis and PXRD. Figure 2 displays the molecular structure of sodium starch glycolate, illustrating the phosphate crosslinking and substitution of hydroxyl by sodium-carboxymethyl groups. Along with the salt impurity levels, which are also characterized here, these are the main attributes that are expected to control the water uptake and dissolution properties of formulations containing these materials. It has been found that by varying the grade of SSG, the water uptake and dissolution properties of formulations changed, though the underlying cause was not found. ${ }^{4}$

Phosphate groups lead to cross-linking between SSG chains, reducing the water-soluble fraction of the polymer, although these effects have been studied very little in the literature. Table 1 gives the sample description and elemental analysis $(\mathrm{P}, \mathrm{Na}$ and $\mathrm{Cl}$ ) results for the SSG samples. The total phosphorus 
content varies by almost a factor of 3 between the samples, it being highest for Source A and Source B, with Source C, Source E and then Source D having the lowest values. The total phosphorus content has contributions from both cross-linked and free phosphorus, the latter being an impurity carried over from the synthesis of the SSG. The two contributions can be distinguished using IR spectroscopy, shown in Figure 3, where the presence of free phosphate $\left(\mathrm{P}-\mathrm{H}_{2} \mathrm{O}\right)$ is observed only in the Source E and Source C samples. This indicates that the phosphorus in the other three samples can be entirely attributed to crosslinked phosphorus, although from the IR results alone it is not possible to further refine the exact amount of cross-linked phosphorus in the Source E or Source C samples. Table 2 includes a summary of the quantification of phosphorus cross-linking. It is interesting to note that Source A and Source B contain the highest overall phosphorus levels and still do not contain any free phosphate.

Sodium-carboxymethyl (-COONa) groups partially replace the hydroxyl (-OH) groups in the SSG and restrict the hydrogen-bond formation of the $\mathrm{OH}$ groups. The extent of carboxymethylation of the hydroxyl groups is estimated through the total sodium content in the different SSGs (Table 1), although this consists of contributions from the Na-carboxymethyl group as well as from salt impurities such as $\mathrm{NaCl}$ and sodium citrate. Indeed, Figure 4 shows the PXRD pattern of a trisodium citrate dihydrate reference in comparison to the PXRD patterns of different sodium starch glycolate samples, confirming the presence of $\mathrm{NaCl}$ impurities in all of the samples $\left(2 \theta \sim 27.5^{\circ}\right.$ and $\left.\sim 31.7^{\circ}\right)$. Trisodium citrate dihydrate was also found as an impurity in the Source C and Source E samples. Quantification of the PXRD data suggests that Source C and Source E contain approximately 4-5 weight \% trisodium citrate dihydrate. PXRD together with elemental analysis was used to quantify the amount of Nacarboxymethyl groups and salt impurities such as sodium chloride and sodium citrate as described. Na associated with $\mathrm{NaCl}$ was calculated from the elemental analysis results of $\mathrm{Cl}$, assuming all of the $\mathrm{Cl}$ to be associated with $\mathrm{Na}$. This allowed $\mathrm{Na}$ associated with the Na-carboxymethyl group to be calculated by subtracting the $\mathrm{Na}$ associated with $\mathrm{NaCl}$ and $\mathrm{Na}$ citrate from the total $\mathrm{Na}$ content from elemental analysis. The results (Table 2) show that Source C and Source E have the highest overall salt content (sum of $\mathrm{NaCl}$ and $\mathrm{Na}$ citrate) and therefore are expected to have the highest ionic strength. Combining elemental analysis results with the PXRD quantification shows that Source E has the most Nacarboxymethylation compared to the rest of the SSGs (Table 2).

The characterization data shows that there is large variability in the intrinsic structure and functionality of SSGs with varying grades, even though all of the samples studied are classified as Type A. These differences could potentially impact the behavior of the different SSG samples in pharmaceutical 
formulations. Increasing the amounts of phosphorus cross-linking and the extent of hydrophobic carboxymethyl groups in the SSG should have opposite effects in drug dissolution behavior. The SSG samples are predominated by phosphorus cross-linking, which goes in the order, Source A and Source B $>$ Source C and Source E > Source D. Source A and Source B contain the most phosphorus content and the most phosphorus cross-linking as all the phosphorus are cross-linked. Source $\mathrm{C}$ and Source E with a higher phosphorus content than Source D has only partial phosphorus cross-linking which could make them behave more like Source D in terms of phosphorus cross-linking. On the other hand, hydrophobic carboxymethyl groups disrupt the formation of hydrogen bonds and cross-linking, allowing water access into the polymer. Source E has the most Na-carboxymethylation. Some of the SSG samples contain salt impurities such as sodium chloride and/or sodium citrate, which can also allow rapid access of water into the polymer, facilitating dissolution. An optimum balance between carboxymethylation, crosslinking and salt impurities allows rapid water uptake and swelling without resulting in high viscosity that would delay dissolution. Information correlating the intrinsic structure and characteristics of the SSG molecule with its behavior would be valuable to a formulator and would help in choosing the right disintegrant, giving insights into optimized drug development. However, the complexity of the structure and functionality of SSG makes this sort of correlation tedious. Moreover, visual correlations of their behavior might not be accurate because macroscopic information obtained does not necessarily reflect the important microscopic processes occurring.

In the second part of this study, the disintegration and water uptake rates of a series of SSG compacts were monitored and quantified using high-resolution, real-time FLASH $^{6-8}$ MRI. The resulting disintegration/water uptake rates were then correlated to the physicochemical characteristics of the SSG samples. Figure 5 demonstrates the image quality and resolution achieved by the MRI and the different stages of hydration/water uptake of the different SSGs. Real time MRI movies are given in the supporting information. By applying the voxel analysis routine to the images for each series, a time resolved estimate of the intact volume of the tablets is obtained. Figure 6 (a) shows these estimates and fits of the curves to estimate the rate of hydration of each SSG compact, while Figure 6 (b) correlates the disintegration time of the compacts/tablets with the $\mathrm{P}$ content/P cross-linking. The quantification of the water uptake rate results shows that the rate of hydration is fastest for Source A and Source B. Source C and Source D have the slowest rate and Source E has an intermediate rate of water uptake. There appears to be a relatively strong positive correlation between these measured disintegration rates and the amount of phosphorus cross-linking. Source A and Source B with the highest phosphorus cross-linking, 
have fast rates of hydration, while Source C and Source D with lower phosphorus cross-linking have the slowest rate of hydration. It should be noted that Source $\mathrm{C}$ with a higher phosphorus content than Source D has only partial phosphorus cross-linking making it behave more like Source D. This correlation is counter-intuitive because cross-linking is expected to reduce the water-soluble fraction of the polymer, leading to insolubility and restricted water access. However, it appears that the phosphorus cross-linking facilitates the formation of an optimal disintegrant that counteracts the excess hydrophilic effect of the other functionalities and salt content. Even though carboxymethylation of the hydroxyl groups reduces the cross-linking and increases the water access to the molecule, the intrinsic hydrophobicity of the carboxymethyl groups could also have an effect on the water uptake. In addition, it should be noted that cross-correlations could also exist.

Phosphorus cross-linking, Na-carboxymethylation and the levels of salt impurities in these materials were found to have large variability within the Type A grade of SSG samples. It should be noted that phosphorus cross-linking and the levels of salt impurities have been studied in depth for the first time. MRI results, together with the characterization data, show that the variability in structure and functionality has an impact on the water uptake and disintegration rates of the SSGs, and therefore, their performance in a formulation. Changes in the hydration rates could also have an impact on the dissolution rates of a drug in a formulation. Obtaining an understanding of such structure-performance correlations is becoming increasingly important to formulators in order to make optimized formulations that can account for the complexity of structure and functionality in these materials. Such information will be valuable to the regularity agencies and FDA during the drug approval process. This relatively simple and robust approach could be used to understand and correlate structure and performance in a variety of materials used as pharmaceutical excipients, with the potential to implement it as a highthroughput technique.

\section{Conclusions}

A complete characterization of a series of commercial sodium starch glycolate (SSG) has been performed, leading to the understanding of their functionalities such as carboxymethylation, phosphorus cross-linking and salt impurities, and showing the variability in their physicochemical properties. A multi-slice FLASH MRI approach in combination with a robust voxel-analysis routine has been used to extract disintegrations rates of SSG compacts. This simple and robust method can reliably estimate 
disintegration rates, giving resolution between tablets that disintegrate at similar rates. A positive correlation has been made between the disintegration rates of SSG compacts and their extent of phosphorus cross-linking, giving insights into excipient selection. SSG grades with higher phosphorus cross-linking were found to have faster water uptake and disintegration rates. Such insights into the correlations between structure and performance are important to formulators, helping to optimize formulations by considering the complexity of the physicochemical nature these materials.

\section{Acknowledgements}

The authors would like to acknowledge Duohai Pan for helping with the IR spectral interpretation. The authors would also like to thank the US National Science Foundation, grant no. CHE 1412064 for MRI technique development in the paper. 
Figure 1: Illustration of the image-processing scheme on a single slice from the Source $C$ series. Top: raw image. Bottom: the same images post-processing, showing the voxels assigned to tablet in pink, dissolution medium in yellow and external regions in black.

Figure 2: Molecular structure of sodium starch glycolate (SSG) showing phosphate cross-linking and sodium-carboxymethyl (-COONa) groups.

Figure 3: IR spectra of different sodium starch glycolate namely, Source A, Source B, Source C, Source D and Source E (top to bottom).

Figure 4: PXRD patterns of different sodium starch glycolate namely, Source A, Source B, Source C, Source D and Source E. Reference PXRD pattern of trisodium citrate is the top pattern. $\mathrm{NaCl}$ is indicated by the arrows $\left(2 \theta\right.$ around $\sim 27.5^{\circ}$ and $\left.\sim 31.7^{\circ}\right)$.

Figure 5: A comparison of the hydration of the SSG compacts by MRI showing a single slice from similar times during the disintegration process. The black region in the tube represents the dry compact. MRI videos can be accessed online in the Supplementary Information.

Figure 6: Quantification of the rate of hydration of the SSG samples by MRI: (a) examples of the fits of the voxel data for each series (b) trend in the fitted hydration rates compared to the phosphorus content of each SSG. Error bars represent the statistical error associated with fitting. 


\section{References}

1. Soumya, M.; Chowdary, Y. A.; Madhuri, A.; Sindhusha, M. D.; Nagarani, T.; Aruna, B.; Manasa, V., Formulation and Invirtoevaluation of fast dissolving tablets of flecainide acetate. Int $J$ Pharm Pharm Sci. 2013, 5 (2).

2. Zhao, N.; Augsburger, L. L., Functionality Comparison of 3 Classes of Superdisintegrants in Promoting Aspirin Tablet Disintegration and Dissolution. AAPS PharmSciTech 2005, 6 (4), E634.

3. Edge, S.; Steele, D. F.; Staniforth, J. N.; Chen, A.; Woodcock, P. M., Powder compaction properties of sodium starch glycolate disintegrants. Drug development and industrial pharmacy 2002, 28 (8), 989-99.

4. Rudnic, E. M.; Kanig, J. L.; Rhodes, C. T., Effect of Molecular Structure Variation on the Disintegrant Action of Sodium Starch Glycolate. Journal of pharmaceutical sciences 1985, 74 (6), 647.

5. Desai, P. M.; Liew, C. V.; Heng, P. W., Understanding disintegrant action by visualization. Journal of pharmaceutical sciences 2012, 101 (6), 2155-64.

6. Quodbach, J.; Kleinebudde, P., A new apparatus for real-time assessment of the particle size distribution of disintegrating tablets. Journal of pharmaceutical sciences 2014, 103 (11), 3657-65.

7. Quodbach, J.; Moussavi, A.; Tammer, R.; Frahm, J.; Kleinebudde, P., Assessment of disintegrant efficacy with fractal dimensions from real-time MRI. International journal of pharmaceutics 2014, 475 (1-2), 605-12.

8. Quodbach, J.; Moussavi, A.; Tammer, R.; Frahm, J.; Kleinebudde, P., Tablet Disintegration Studied by High-Resolution Real-Time Magnetic Resonance Imaging. Journal of pharmaceutical sciences 2014, 103 (1), 249-255.

9. Abrahmsen-Alami, S.; Korner, A.; Nilsson, I.; Larsson, A., New release cell for NMR microimaging of tablets. Swelling and erosion of poly(ethylene oxide). International journal of pharmaceutics 2007, 342 (1-2), 105-14.

10. Chen, Y. Y.; Hughes, L. P.; Gladden, L. F.; Mantle, M. D., Quantitative ultra-fast MRI of HPMC swelling and dissolution. Journal of pharmaceutical sciences 2010, 99 (8), 3462-72.

11. Djemai, A.; Sinka, I. C., NMR imaging of density distributions in tablets. International journal of pharmaceutics 2006, 319 (1-2), 55-62.

12. Ellison, C. D.; Ennis, B. J.; Hamad, M. L.; Lyon, R. C., Measuring the distribution of density and tabletting force in pharmaceutical tablets by chemical imaging. Journal of pharmaceutical and biomedical analysis 2008, 48 (1), 1-7.

13. Ferrero, C.; Massuelle, D.; Jeannerat, D.; Doelker, E., Towards elucidation of the drug release mechanism from compressed hydrophilic matrices made of cellulose ethers. I. Pulse-field-gradient spinecho NMR study of sodium salicylate diffusivity in swollen hydrogels with respect to polymer matrix physical structure. Journal of controlled release : official journal of the Controlled Release Society 2008, 128 (1), 71-9.

14. Knoos, P.; Topgaard, D.; Wahlgren, M.; Ulvenlund, S.; Piculell, L., Using NMR chemical shift imaging to monitor swelling and molecular transport in drug-loaded tablets of hydrophobically modified poly(acrylic acid): methodology and effects of polymer (in)solubility. Langmuir : the ACS journal of surfaces and colloids 2013, 29 (45), 13898-908.

15. Kulinowski, P.; Dorozynski, P.; Jachowicz, R.; Weglarz, W. P., An integrated system for dissolution studies and magnetic resonance imaging of controlled release, polymer-based dosage formsa tool for quantitative assessment of hydrogel formation processes. Journal of pharmaceutical and biomedical analysis 2008, 48 (3), 685-93.

16. Mikac, U.; Demsar, A.; Demsar, F.; Sersa, I., A study of tablet dissolution by magnetic resonance electric current density imaging. Journal of magnetic resonance 2007, 185 (1), 103-9. 
17. Mikac, U.; Sepe, A.; Kristl, J.; Baumgartner, S., A new approach combining different MRI methods to provide detailed view on swelling dynamics of xanthan tablets influencing drug release at different $\mathrm{pH}$ and ionic strength. Journal of controlled release : official journal of the Controlled Release Society 2010, 145 (3), 247-56.

18. Nott, K. P., Magnetic resonance imaging of tablet dissolution. European journal of pharmaceutics and biopharmaceutics : official journal of Arbeitsgemeinschaft fur Pharmazeutische Verfahrenstechnik e.V 2010, 74 (1), 78-83.

19. Richardson, J. C.; Bowtell, R. W.; Mader, K.; Melia, C. D., Pharmaceutical applications of magnetic resonance imaging (MRI). Advanced drug delivery reviews 2005, 57 (8), 1191-209.

20. Baille, W. E.; Malveau, C. d.; Zhu, X. X.; Marchessault, R. H., NMR Imaging of High-Amylose Starch Tablets. 1. Swelling and Water Uptake. Biomacromolecules 2002, 3, 214.

21. Melia, C. D.; Rajabi-Siahboomi, A. R.; Bowtell, R. W., Magnetic resonance imaging of controlled release pharmaceutical dosage forms. Pharmaceutical Science \& Technology Today 1998, 1 , 1461.

22. Haase, A.; Frahm, J.; Matthaei, D.; Hanicke, W.; Merboldt, K.-D., FLASH Imaging. Rapid NMR Imaging Using Low Flip-Angle Pulses. Journal of magnetic resonance 1986, 67, 258. 
Table 1: Sample description and elemental analysis of different sodium starch glycolate (Type A) namely, Source A, Source B, Source C, Source D and Source E.

\begin{tabular}{|l|l|l|l|l|l|l|}
\hline Me-OH based & Et-OH based & $\mathbf{P}(\mathbf{p p m})$ & $\mathbf{N a}(\%)$ & $\mathbf{C l}(\%)$ & pH value & Type \\
\hline & Source A & 1350 & 4.8 & 2.04 & \\
\hline Source B & 1150 & 4.88 & 2.17 & \\
\hline & Source C & 720 & 5.75 & 2.81 & $5.5-7.5$ & A \\
\hline & Source D & Source E & 740 & 7.24 & 2.70 & \\
\hline
\end{tabular}


Table 2: The quantification of phosphorus cross-linking, Na-carboxymethyl groups, $\mathrm{NaCl}$ and $\mathrm{Na}$ citrate salt impurities of sodium starch glycolate (Type A) namely, Source A, Source B, Source C, Source D and Source E.

\begin{tabular}{|c|c|c|c|c|}
\hline SSG samples & P cross-linking & $\begin{array}{c}\mathrm{Na}(\%) \\
\text { Carboxy } \\
\text { methylation }\end{array}$ & $\begin{array}{c}\mathbf{N a ( \% )} \\
\mathrm{NaCl}\end{array}$ & $\begin{array}{c}\mathrm{Na}(\%) \\
\mathrm{Na} \text { citrate }\end{array}$ \\
\hline Source A & all & 3.48 & 1.32 & 0 \\
\hline Source B & all & 3.47 & 1.41 & 0 \\
\hline Source C & partial & 2.93 & 1.82 & $0.94-1.17 \%$ \\
\hline Source D & all & 3.1 & 1.61 & 0 \\
\hline Source E & partial & 4.49 & 1.75 & $0.94-1.17 \%$ \\
\hline
\end{tabular}


Figures

Figure 1

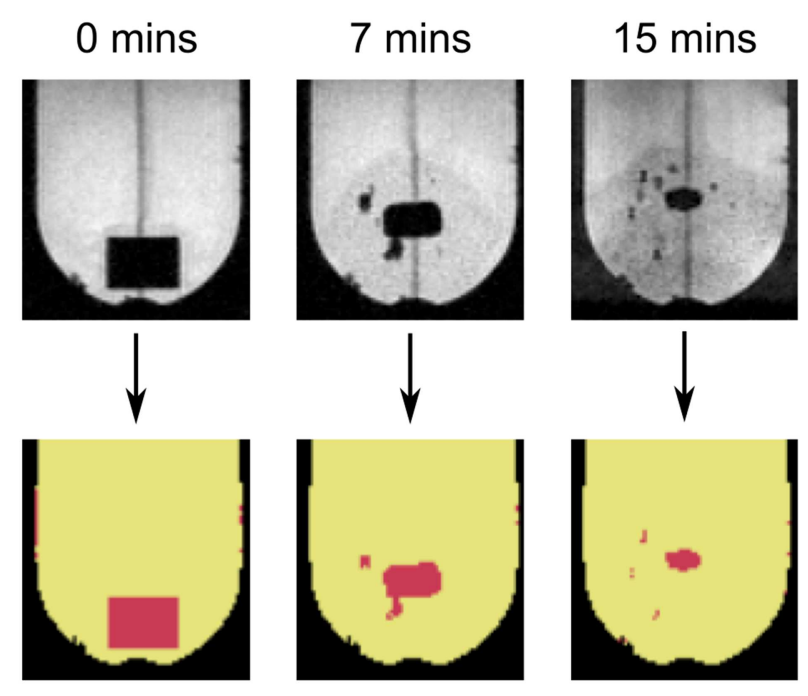


Figure 2

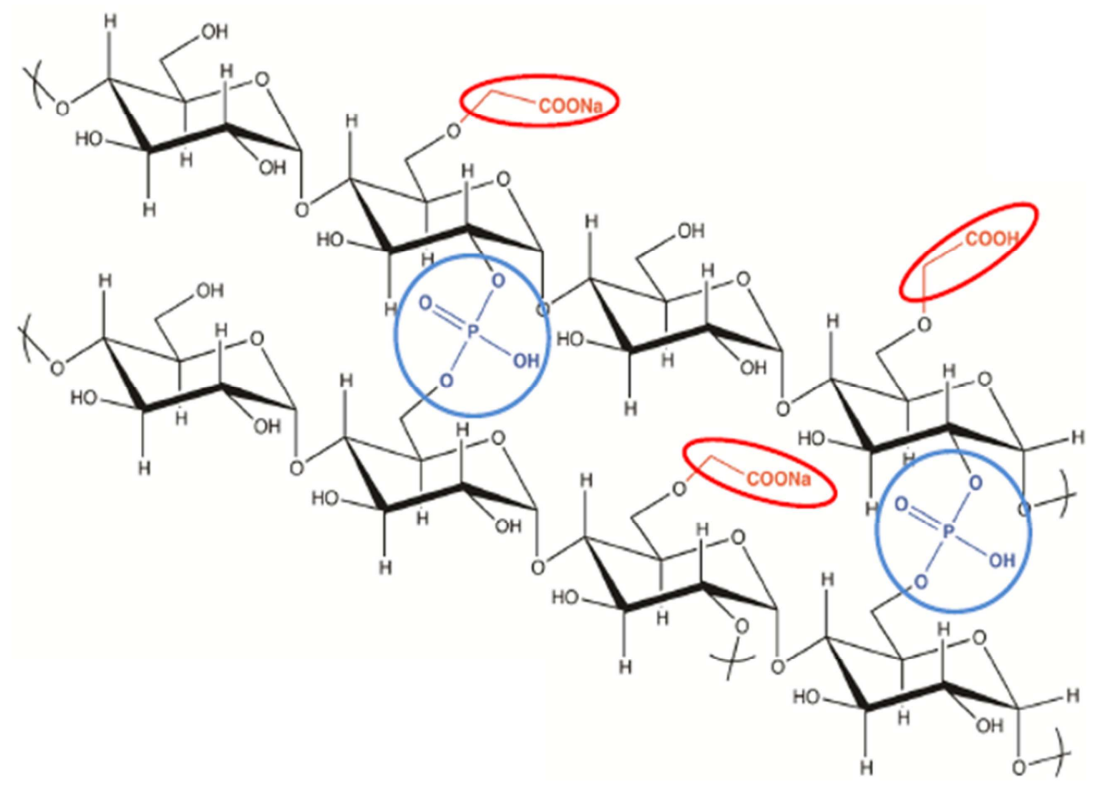




\section{Figure 3}

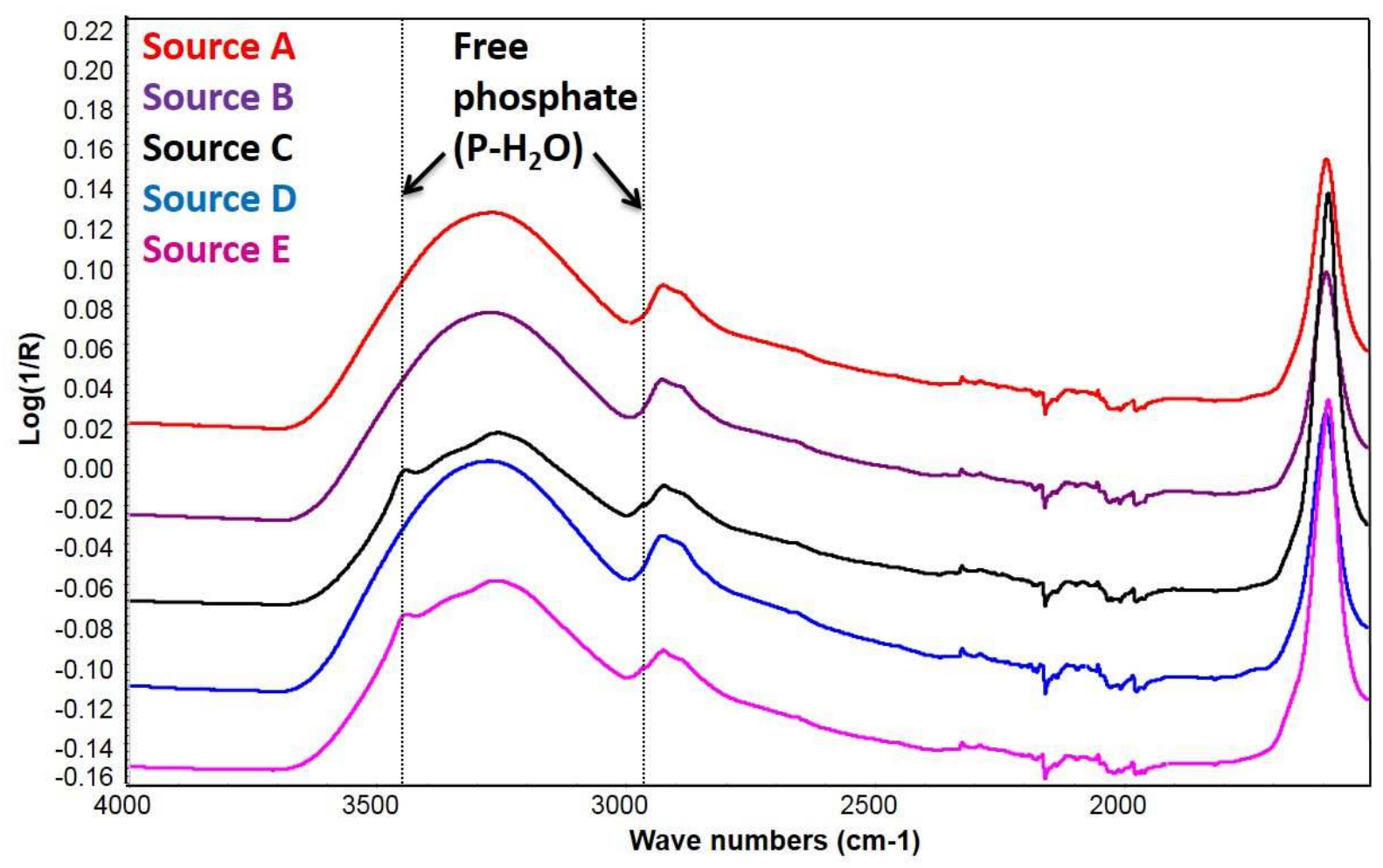


Figure 4

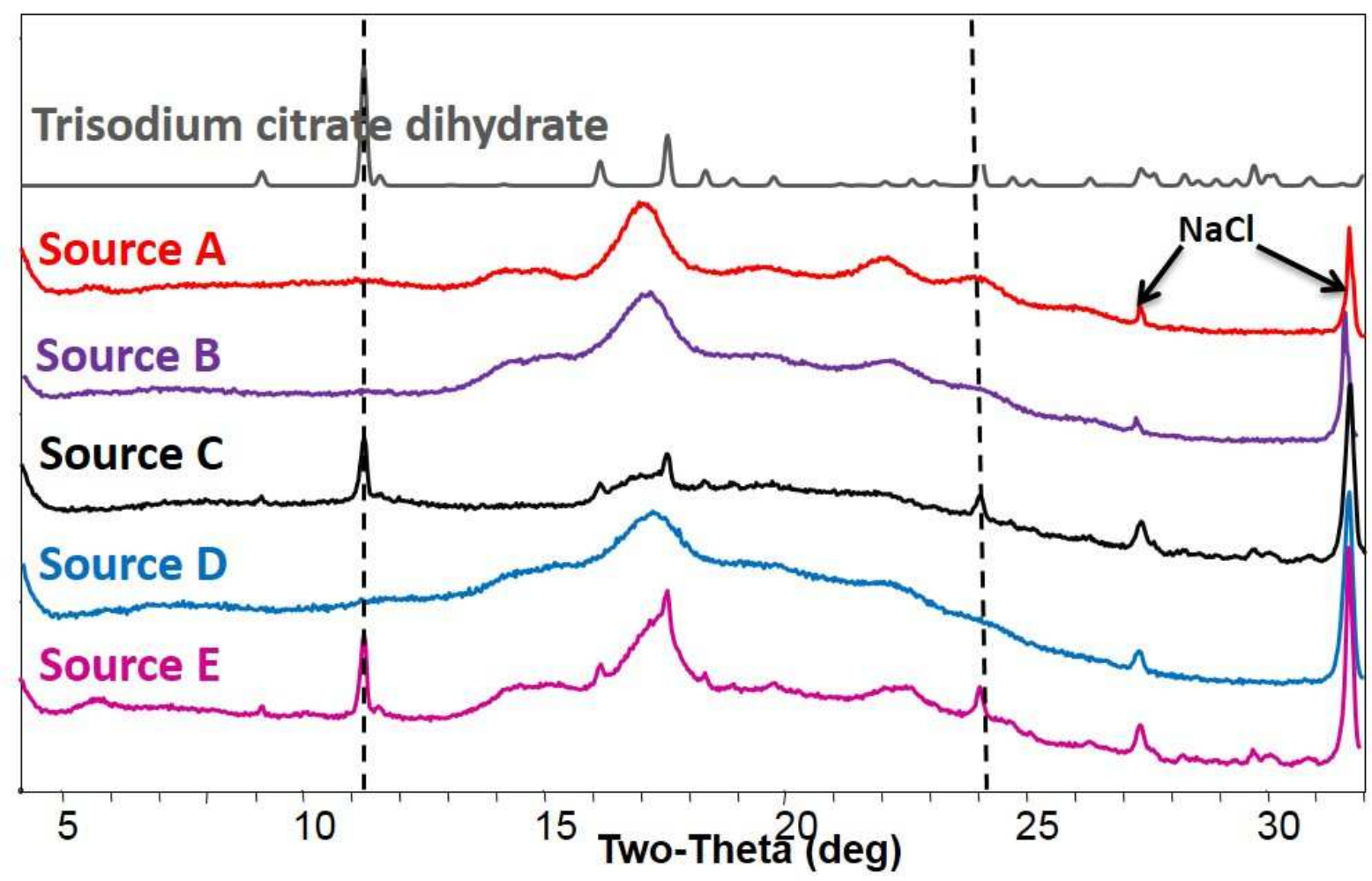


Figure 5

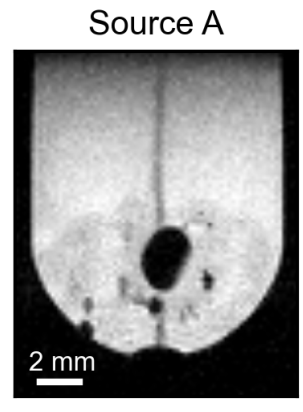

Time: $171 \mathrm{~s}$
Source B

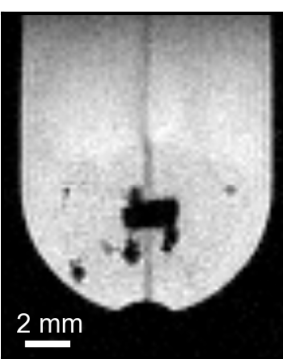

Time: $177 \mathrm{~s}$
Source C

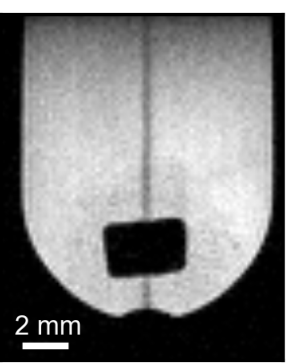

Time: $172 \mathrm{~s}$
Source D

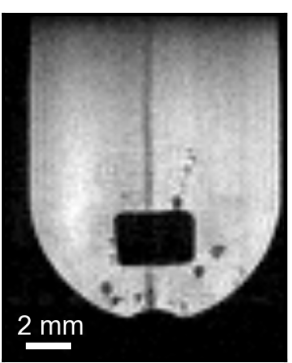

Time: $180 \mathrm{~s}$
Source E

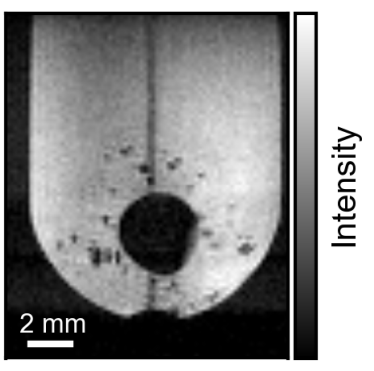

Time: $178 \mathrm{~s}$ 
Figure 6

(a)

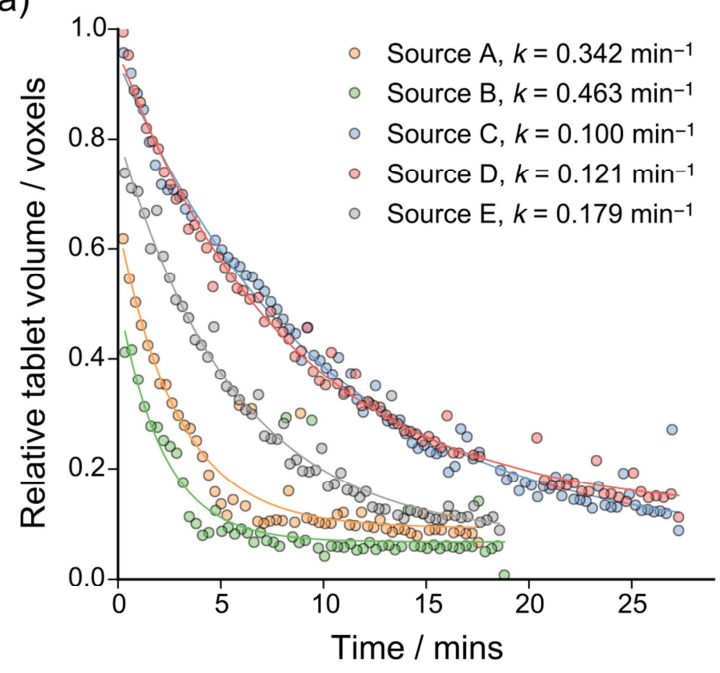

(b)

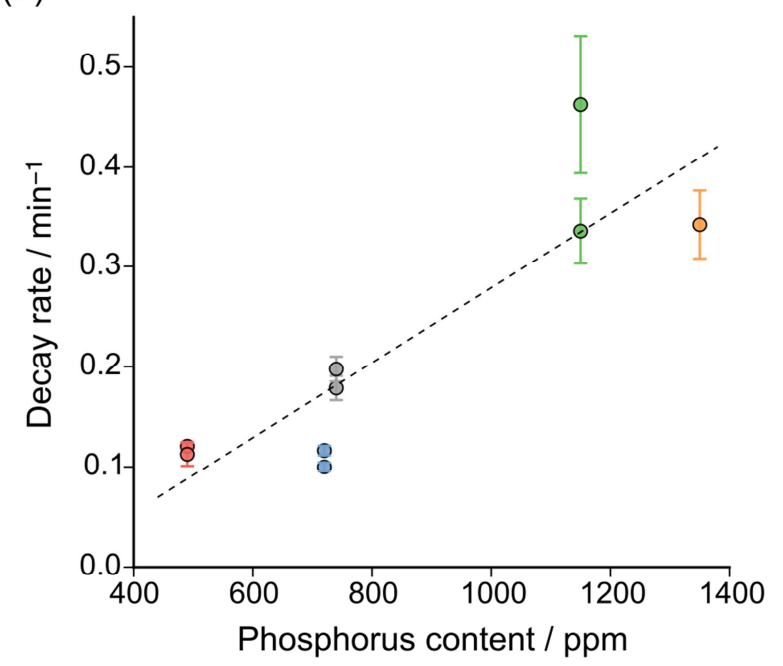

